\title{
MULTI-FACTOR FINANCIAL DERIVATIVES ON FINITE DOMAINS*
}

\author{
YOU-LAN ZHU ${ }^{\dagger}$ AND JINLIANG (ERIC) $\mathrm{LI}^{\ddagger}$
}

\begin{abstract}
In this paper, we introduce reversion conditions for stochastic models. Also we prove that if the models satisfy reversion conditions and the market prices of risks are bounded, then the final-value problem of general two-factor financial derivative equations on rectangular domains has a unique solution. For such problems we can obtain their numerical solutions without using any artificial conditions. Examples show that if the singularity-separating method and extrapolation techniques are used, then very good solutions can be obtained even on very coarse meshes.
\end{abstract}

\section{Introduction}

In finance, a stochastic model usually describes a random variable which can take its value on an infinite domain. Using such a model, many problems of financial derivative pricing can be written as parabolic problems defined on an infinite domain. One usual way to obtain numerical solutions of such problems is to solve the problem on a large finite domain. Since parabolic problems on a finite domain need boundary conditions, some approximate boundary conditions on artificial boundaries need to be added (see [4]). In this case, we have to make sure that the formulation of the approximate problem is well-posed and that the error introduced by the approximate boundary conditions is small enough so that the accuracy of numerical solutions is not affected. Can we have stochastic models which describe random variables on finite domains so that such problems can be avoided? In [5], for the one dimensional case, we provided so-called reversion conditions ${ }^{1)}$ under which parabolic equations in finance degenerate, and proved that these conditions are sufficient and necessary for an initial-value problem of corresponding degenerate parabolic equations on finite domains to have a unique solution. In this paper, for the two-dimensional case, we will give some conditions and prove that under such conditions a final-value problem $^{2}$ ) of corresponding degenerate parabolic equations on finite domains has a unique solution $^{3)}$, and provide examples to explain that numerical solutions of such final-value problems can be obtained by the degenerate parabolic partial differential equation and final condition; that is, no artificial boundary conditions are needed.

The rest of this paper is organized as follows. In the next section, we introduce the reversion conditions and show how a model can be modified so that the reversion conditions are satisfied. In Section 3, we prove that the solution of a final-value problem of corresponding degenerate parabolic equations on finite domains is unique if the stochastic models satisfy reversion conditions. Then, we apply the result to the options on assets with stochastic volatilities and show that such a two-factor European option problem is a final-value problem on a finite domain and has a unique solution if the stochastic model for the volatility satisfies the reversion conditions. In Section 5 , a numerical method without using any artificial conditions and numerical results

\footnotetext{
* Received: November 12, 2002; accepted (in revised version): January 10, 2003.

$\dagger$ Dept. of Mathematics, University of North Carolina at Charlotte, NC. (yzhu@math.uncc.edu).

¥ Quantitative Research US, Bank of America. (eric.li@bankofamerica.com).

1) Similar conditions have also been given in [3].

2) In finance, the problems are usually final-value problems of parabolic partial differential equations, and those problems can be easily converted into initial-value problems.

3) Even though we give the complete proof only for the two-dimensional case, the proof for higher dimensional cases is outlined.
} 
for this final-value problem on a finite domain are given. We also show that if the singularity-separating method and extrapolation techniques are used, then numerical solutions are very good even on quite coarse meshes. Finally, our conclusions are given.

\section{Reversion Conditions for Stochastic Models}

Suppose that a financial derivative depends on the time $t$ and two random variables $S_{1}$ and $S_{2}$, and that $S_{1}$ and $S_{2}$ follow

$$
d S_{1}=a_{1}\left(S_{1}, S_{2}, t\right) d t+b_{1}\left(S_{1}, S_{2}, t\right) d X_{1},
$$

and

$$
d S_{2}=a_{2}\left(S_{1}, S_{2}, t\right) d t+b_{2}\left(S_{1}, S_{2}, t\right) d X_{2},
$$

where $d X_{1}$ and $d X_{2}$ are two Wiener processes. They are correlated and $\mathrm{E}\left[d X_{1} d X_{2}\right]=$ $\rho d t$, where $|\rho| \leq 1$. Such financial derivatives are referred to as two-factor ones in this paper.

In real life, $S_{1}$ and $S_{2}$ usually have lower and upper bounds. Let $S_{i l}, S_{i u}$ be the lower and upper bounds of $S_{i}$ respectively, $i=1,2$. In this paper for $S_{i}$ we require the following so-called reversion conditions:

$$
\left\{\begin{array}{l}
{\left.\left[a_{i}\left(S_{1}, S_{2}, t\right)-b_{i}\left(S_{1}, S_{2}, t\right) \frac{\partial b_{i}\left(S_{1}, S_{2}, t\right)}{\partial S_{i}}\right]\right|_{S_{i}=S_{i l}} \geq 0} \\
\left.b_{i}\left(S_{1}, S_{2}, t\right)\right|_{S_{i}=S_{i l}}=0
\end{array}\right.
$$

and

$$
\left\{\begin{array}{l}
{\left.\left[a_{i}\left(S_{1}, S_{2}, t\right)-b_{i}\left(S_{1}, S_{2}, t\right) \frac{\partial b_{i}\left(S_{1}, S_{2}, t\right)}{\partial S_{i}}\right]\right|_{S_{i}=S_{i u}} \leq 0} \\
\left.b_{i}\left(S_{1}, S_{2}, t\right)\right|_{S_{i}=S_{i u}}=0
\end{array}\right.
$$

Here conditions (2.3) and (2.4) for $S_{1}$ should hold for all $S_{2} \in\left[S_{2 l}, S_{2 u}\right]$ and conditions (2.3) and (2.4) for $S_{2}$ should hold for all $S_{1} \in\left[S_{1 l}, S_{1 u}\right]$. It is clear that if $\left.\frac{\partial b_{i}\left(S_{1}, S_{2}, t\right)}{\partial S_{i}}\right|_{S_{i}=S_{i l}}$ and $\left.\frac{\partial b_{i}\left(S_{1}, S_{2}, t\right)}{\partial S_{i}}\right|_{S_{i}=S_{i u}}$ are bounded, then the two conditions (2.3) and (2.4) can be reduced to

$$
\left\{\begin{array}{l}
\left.a_{i}\left(S_{1}, S_{2}, t\right)\right|_{S_{i}=S_{i l}} \geq 0 \\
\left.b_{i}\left(S_{1}, S_{2}, t\right)\right|_{S_{i}=S_{i l}}=0
\end{array}\right.
$$

and

$$
\left\{\begin{array}{l}
\left.a_{i}\left(S_{1}, S_{2}, t\right)\right|_{S_{i}=S_{i u}} \leq 0 \\
\left.b_{i}\left(S_{1}, S_{2}, t\right)\right|_{S_{i}=S_{i u}}=0
\end{array}\right.
$$

In finance, if a model satisfies the inequality conditions in (2.5) and (2.6), then it is said that the model has the property of mean reverting. However, if the equality conditions in (2.5) and (2.6) do not hold, then there is still a chance for the random variable to go to infinity. It is easy to modify those models so that they satisfy all the reversion conditions. For example, suppose that for $S_{2}$ the equality conditions in 
reversion conditions do not hold. We can modify the model for $S_{2}$ as follows: the coefficient of $d X_{2}$ is multiplied by a function, for example,

$$
\Phi(x)=\frac{1-(1-2 x)^{2}}{1-0.975(1-2 x)^{2}},
$$

where $x=\frac{\left(S_{2}-S_{2 l}\right)}{\left(S_{2 u}-S_{2 l}\right)}$. Because $\Phi(x)$ are equal to zero at $S_{2}=S_{2 l}$ and $S_{2}=S_{2 u}$ and very close to one at $S_{2} \in\left(S_{2 l}, S_{2 u}\right)$, almost all the properties of the original model are kept and the reversion conditions will hold after the modification is made. In the next section we are going to explain that if (2.3) and (2.4) hold, then the two random variables $S_{1}$ and $S_{2}$ are guaranteed to be in $\left[S_{1 l}, S_{1 u}\right]$, $\left[S_{2 l}, S_{2 u}\right]$ respectively.

\section{Uniqueness of Solutions for Final-Value Problems}

Suppose that a financial derivative depends on the time $t$ and two random variables $S_{1}$ and $S_{2}$, which satisfy (2.1) and (2.2) and the reversion conditions, and let $V\left(S_{1}, S_{2}, t\right)$ be the price of the financial derivative. By arbitrage argument, it can be shown that $V\left(S_{1}, S_{2}, t\right)$ should satisfy the following equation (see [8]):

$$
\begin{aligned}
& \frac{\partial V}{\partial t}+\frac{1}{2} b_{1}^{2} \frac{\partial^{2} V}{\partial S_{1}^{2}}+\rho b_{1} b_{2} \frac{\partial^{2} V}{\partial S_{1} \partial S_{2}} \\
+ & \frac{1}{2} b_{2}^{2} \frac{\partial^{2} V}{\partial S_{2}^{2}}+\left(a_{1}-\lambda_{1} b_{1}\right) \frac{\partial V}{\partial S_{1}}+\left(a_{2}-\lambda_{2} b_{2}\right) \frac{\partial V}{\partial S_{2}}-r V=0,
\end{aligned}
$$

where $\lambda_{1}$ and $\lambda_{2}$ are two bounded functions and called market prices of risk on $S_{1}$ and $S_{2}$ respectively, and $r$ is the spot interest rate. Also, many financial derivatives should be solutions of the final-value problem

$$
\left\{\begin{array}{c}
\frac{\partial V}{\partial t}+\frac{1}{2} b_{1}^{2} \frac{\partial^{2} V}{\partial S_{1}^{2}}+\rho b_{1} b_{2} \frac{\partial^{2} V}{\partial S_{1} \partial S_{2}}+\frac{1}{2} b_{2}^{2} \frac{\partial^{2} V}{\partial S_{2}^{2}} \\
+\left(a_{1}-\lambda_{1} b_{1}\right) \frac{\partial V}{\partial S_{1}}+\left(a_{2}-\lambda_{2} b_{2}\right) \frac{\partial V}{\partial S_{2}}-r V=0, \\
S_{1} \in\left[S_{1 l}, S_{1 u}\right], \quad S_{2} \in\left[S_{2 l}, S_{2 u}\right], \quad t \in[0, T], \\
V\left(S_{1}, S_{2}, T\right)=f\left(S_{1}, S_{2}\right), \quad S_{1} \in\left[S_{1 l}, S_{1 u}\right], \quad S_{2} \in\left[S_{2 l}, S_{2 u}\right] .
\end{array}\right.
$$

Now let us discuss when problem (3.1) has a unique solution. For this question, we have the following theorem:

THEOREM 3.1. If

(i). the reversion conditions (2.3) and (2.4) hold;

(ii). there exists a constant $c_{1}$ such that

$$
\begin{aligned}
\max _{\substack{S_{1 l} \leq S_{1} \leq S_{1 u} \\
S_{2 l} \leq S_{2} \leq S_{2 u}}} \mid \frac{\partial}{\partial S_{1}}\left(a_{1}-\lambda_{1} b_{1}-b_{1} \frac{\partial b_{1}}{\partial S_{1}}-\frac{1}{2} \frac{\partial}{\partial S_{2}}\left(\rho b_{1} b_{2}\right)\right) \\
+\frac{\partial}{\partial S_{2}}\left(a_{2}-\lambda_{2} b_{2}-b_{2} \frac{\partial b_{2}}{\partial S_{2}}-\frac{1}{2} \frac{\partial}{\partial S_{1}}\left(\rho b_{1} b_{2}\right)\right)+2 r \mid \leq c_{1} ; \text { and }
\end{aligned}
$$

(iii). solutions of problem (3.1) exist and their first derivatives are bounded, then the solution of (3.1) is unique. 
Proof. Suppose that $u_{1}$ and $u_{2}$ are two solutions of (3.1) and let $u=u_{1}-u_{2}$. It is clear that $u$ is the solution of the problem (3.1) with $f\left(S_{1}, S_{2}\right) \equiv 0$. Let $\tau=T-t$ and define

$$
W(\tau)=\int_{S_{2 l}}^{S_{2 u}} \int_{S_{1 l}}^{S_{1 u}} u^{2}\left(S_{1}, S_{2}, T-\tau\right) d S_{1} d S_{2} .
$$

Since the partial differential equation in (3.1) can be rewritten as

$$
\begin{aligned}
\frac{\partial u}{\partial \tau}= & \frac{1}{2} \frac{\partial}{\partial S_{1}}\left(b_{1}^{2} \frac{\partial u}{\partial S_{1}}+\rho b_{1} b_{2} \frac{\partial u}{\partial S_{2}}\right)+\frac{1}{2} \frac{\partial}{\partial S_{2}}\left(\rho b_{1} b_{2} \frac{\partial u}{\partial S_{1}}+b_{2}^{2} \frac{\partial u}{\partial S_{2}}\right) \\
& +\left(a_{1}-\lambda_{1} b_{1}-b_{1} \frac{\partial b_{1}}{\partial S_{1}}-\frac{1}{2} \frac{\partial}{\partial S_{2}}\left(\rho b_{1} b_{2}\right)\right) \frac{\partial u}{\partial S_{1}} \\
& +\left(a_{2}-\lambda_{2} b_{2}-b_{2} \frac{\partial b_{2}}{\partial S_{2}}-\frac{1}{2} \frac{\partial}{\partial S_{1}}\left(\rho b_{1} b_{2}\right)\right) \frac{\partial u}{\partial S_{2}}-r u
\end{aligned}
$$

we have

$$
\begin{aligned}
\frac{1}{2} \frac{d W(\tau)}{d \tau}= & \int_{S_{2 l}}^{S_{2 u}} \int_{S_{1 l}}^{S_{1 u}} u \frac{\partial u}{\partial \tau} d S_{1} d S_{2} \\
= & \int_{S_{2 l}}^{S_{2 u}} \int_{S_{1 l}}^{S_{1 u}} \frac{u}{2} \frac{\partial}{\partial S_{1}}\left(b_{1}^{2} \frac{\partial u}{\partial S_{1}}+\rho b_{1} b_{2} \frac{\partial u}{\partial S_{2}}\right) d S_{1} d S_{2} \\
& +\int_{S_{2 l}}^{S_{2 u}} \int_{S_{1 l}}^{S_{1 u}} \frac{u}{2} \frac{\partial}{\partial S_{2}}\left(\rho b_{1} b_{2} \frac{\partial u}{\partial S_{1}}+b_{2}^{2} \frac{\partial u}{\partial S_{2}}\right) d S_{1} d S_{2} \\
& +\int_{S_{2 l}}^{S_{2 u}} \int_{S_{1 l}}^{S_{1 u}} u\left(a_{1}-\lambda_{1} b_{1}-b_{1} \frac{\partial b_{1}}{\partial S_{1}}-\frac{1}{2} \frac{\partial}{\partial S_{2}}\left(\rho b_{1} b_{2}\right)\right) \frac{\partial u}{\partial S_{1}} d S_{1} d S_{2} \\
& +\int_{S_{2 l}}^{S_{2 u}} \int_{S_{1 l}}^{S_{1 u}} u\left(a_{2}-\lambda_{2} b_{2}-b_{2} \frac{\partial b_{2}}{\partial S_{2}}-\frac{1}{2} \frac{\partial}{\partial S_{1}}\left(\rho b_{1} b_{2}\right)\right) \frac{\partial u}{\partial S_{2}} d S_{1} d S_{2} \\
& -\int_{S_{2 l}}^{S_{2 u}} \int_{S_{1 l}}^{S_{1 u}} r u^{2} d S_{1} d S_{2} .
\end{aligned}
$$

Now let us look at the first four terms in the right hand side of (3.3). Using integration by parts and the equality conditions in (2.3) and (2.4), we can rewrite the first and second terms as follows:

$$
\begin{aligned}
& \int_{S_{2 l}}^{S_{2 u}} \int_{S_{1 l}}^{S_{1 u}} \frac{u}{2} \frac{\partial}{\partial S_{1}}\left(b_{1}^{2} \frac{\partial u}{\partial S_{1}}+\rho b_{1} b_{2} \frac{\partial u}{\partial S_{2}}\right) d S_{1} d S_{2} \\
= & \frac{1}{2} \int_{S_{2 l}}^{S_{2 u}}\left\{\left.\left[u\left(b_{1}^{2} \frac{\partial u}{\partial S_{1}}+\rho b_{1} b_{2} \frac{\partial u}{\partial S_{2}}\right)\right]\right|_{S_{1 l}} ^{S_{1 u}}\right. \\
& \left.-\int_{S_{1 l}}^{S_{1 u}}\left(b_{1}^{2} \frac{\partial u}{\partial S_{1}}+\rho b_{1} b_{2} \frac{\partial u}{\partial S_{2}}\right) \frac{\partial u}{\partial S_{1}} d S_{1}\right\} d S_{2} \\
= & -\frac{1}{2} \int_{S_{2 l}}^{S_{2 u}} \int_{S_{1 l}}^{S_{1 u}}\left(b_{1}^{2} \frac{\partial u}{\partial S_{1}}+\rho b_{1} b_{2} \frac{\partial u}{\partial S_{2}}\right) \frac{\partial u}{\partial S_{1}} d S_{1} d S_{2}
\end{aligned}
$$


and

$$
\begin{aligned}
& \int_{S_{2 l}}^{S_{2 u}} \int_{S_{1 l}}^{S_{1 u}} \frac{u}{2} \frac{\partial}{\partial S_{2}}\left(\rho b_{1} b_{2} \frac{\partial u}{\partial S_{1}}+b_{2}^{2} \frac{\partial u}{\partial S_{2}}\right) d S_{1} d S_{2} \\
= & \frac{1}{2} \int_{S_{1 l}}^{S_{1 u}}\left\{\left.\left[u\left(\rho b_{1} b_{2} \frac{\partial u}{\partial S_{1}}+b_{2}^{2} \frac{\partial u}{\partial S_{2}}\right)\right]\right|_{S_{2 l}} ^{S_{2 u}}\right. \\
& \left.-\int_{S_{2 l}}^{S_{2 u}}\left(\rho b_{1} b_{2} \frac{\partial u}{\partial S_{1}}+b_{2}^{2} \frac{\partial u}{\partial S_{2}}\right) \frac{\partial u}{\partial S_{2}} d S_{2}\right\} d S_{1} \\
= & -\frac{1}{2} \int_{S_{2 l}}^{S_{2 u}} \int_{S_{1 l}}^{S_{1 u}}\left(\rho b_{1} b_{2} \frac{\partial u}{\partial S_{1}}+b_{2}^{2} \frac{\partial u}{\partial S_{2}}\right) \frac{\partial u}{\partial S_{2}} d S_{1} d S_{2} .
\end{aligned}
$$

Also according to the equality condition in $(2.3), b_{1}\left(S_{1 l}, S_{2}, t\right)=0$ is equal to zero for any $S_{2}$, so $\left.\frac{\partial}{\partial S_{2}}\left(\rho b_{1} b_{2}\right)\right|_{S_{1}=S_{1 l}}=0$. Similarly, we have $\left.\frac{\partial}{\partial S_{2}}\left(\rho b_{1} b_{2}\right)\right|_{S_{1}=S_{1 u}}=0$, $\left.\frac{\partial}{\partial S_{1}}\left(\rho b_{1} b_{2}\right)\right|_{S_{2}=S_{2 l}}=0$ and $\left.\frac{\partial}{\partial S_{1}}\left(\rho b_{1} b_{2}\right)\right|_{S_{2}=S_{2 u}}=0$. Noticing these facts and the inequality conditions in (2.3) and (2.4), for the third and fourth integrals in the right hand side of (3.3) we have

$$
\begin{aligned}
& \int_{S_{2 l}}^{S_{2 u}} \int_{S_{1 l}}^{S_{1 u}} u\left(a_{1}-\lambda_{1} b_{1}-b_{1} \frac{\partial b_{1}}{\partial S_{1}}-\frac{1}{2} \frac{\partial}{\partial S_{2}}\left(\rho b_{1} b_{2}\right)\right) \frac{\partial u}{\partial S_{1}} d S_{1} d S_{2} \\
=\frac{1}{2} \int_{S_{2 l}}^{S_{2 u}}\left\{\left.\left[u^{2}\left(a_{1}-\lambda_{1} b_{1}-b_{1} \frac{\partial b_{1}}{\partial S_{1}}-\frac{1}{2} \frac{\partial}{\partial S_{2}}\left(\rho b_{1} b_{2}\right)\right)\right]\right|_{S_{1 l}} ^{S_{1 u}}\right. & \left.\quad-\int_{S_{1 l}}^{S_{1 u}} u^{2} \frac{\partial}{\partial S_{1}}\left(a_{1}-\lambda_{1} b_{1}-b_{1} \frac{\partial b_{1}}{\partial S_{1}}-\frac{1}{2} \frac{\partial}{\partial S_{2}}\left(\rho b_{1} b_{2}\right)\right) d S_{1}\right\} d S_{2} \\
\leq & -\frac{1}{2} \int_{S_{2 l}}^{S_{2 u}} \int_{S_{1 l}}^{S_{1 u}} u^{2} \frac{\partial}{\partial S_{1}}\left(a_{1}-\lambda_{1} b_{1}-b_{1} \frac{\partial b_{1}}{\partial S_{1}}-\frac{1}{2} \frac{\partial}{\partial S_{2}}\left(\rho b_{1} b_{2}\right)\right) d S_{1} d S_{2}
\end{aligned}
$$

and

$$
\begin{aligned}
& \int_{S_{1 l}}^{S_{1 u}} \int_{S_{2 l}}^{S_{2 u} u} u\left(a_{2}-\lambda_{2} b_{2}-b_{2} \frac{\partial b_{2}}{\partial S_{2}}-\frac{1}{2} \frac{\partial}{\partial S_{1}}\left(\rho b_{1} b_{2}\right)\right) \frac{\partial u}{\partial S_{2}} d S_{2} d S_{1} \\
= & \frac{1}{2} \int_{S_{1 l}}^{S_{1 u}}\left\{\left.\left[u^{2}\left(a_{2}-\lambda_{2} b_{2}-b_{2} \frac{\partial b_{2}}{\partial S_{2}}-\frac{1}{2} \frac{\partial}{\partial S_{1}}\left(\rho b_{1} b_{2}\right)\right)\right]\right|_{S_{2 l}} ^{S_{2 u}}\right. \\
& \left.-\int_{S_{2 l}}^{S_{2 u}} u^{2} \frac{\partial}{\partial S_{2}}\left(a_{2}-\lambda_{2} b_{2}-b_{2} \frac{\partial b_{2}}{\partial S_{2}}-\frac{1}{2} \frac{\partial}{\partial S_{1}}\left(\rho b_{1} b_{2}\right)\right) d S_{2}\right\} d S_{1} \\
\leq & -\frac{1}{2} \int_{S_{2 l}}^{S_{2 u}} \int_{S_{1 l}}^{S_{1 u}} u^{2} \frac{\partial}{\partial S_{2}}\left(a_{2}-\lambda_{2} b_{2}-b_{2} \frac{\partial b_{2}}{\partial S_{2}}-\frac{1}{2} \frac{\partial}{\partial S_{1}}\left(\rho b_{1} b_{2}\right)\right) d S_{1} d S_{2} .
\end{aligned}
$$


Adding (3.4) and (3.5) together, due to $|\rho| \leq 1$, we have

$$
\begin{aligned}
& \int_{S_{2 l}}^{S_{2 u}} \int_{S_{1 l}}^{S_{1 u}} \frac{u}{2} \frac{\partial}{\partial S_{1}}\left(b_{1}^{2} \frac{\partial u}{\partial S_{1}}+\rho b_{1} b_{2} \frac{\partial u}{\partial S_{2}}\right) d S_{1} d S_{2} \\
& +\int_{S_{2 l}}^{S_{2 u}} \int_{S_{1 l}}^{S_{1 u}} \frac{u}{2} \frac{\partial}{\partial S_{2}}\left(\rho b_{1} b_{2} \frac{\partial u}{\partial S_{1}}+b_{2}^{2} \frac{\partial u}{\partial S_{2}}\right) d S_{1} d S_{2} \\
= & -\frac{1}{2} \int_{S_{2 l}}^{S_{2 u}} \int_{S_{1 l}}^{S_{1 u}}\left[\left(b_{1} \frac{\partial u}{\partial S_{1}}\right)^{2}+2 \rho b_{1} b_{2} \frac{\partial u}{\partial S_{1}} \frac{\partial u}{\partial S_{2}}+\left(b_{2} \frac{\partial u}{\partial S_{2}}\right)^{2}\right] d S_{1} d S_{2} \leq 0 .
\end{aligned}
$$

Substituting (3.4)-(3.7) into (3.3) and applying (3.8) and condition (ii), we have

$$
\begin{aligned}
\frac{1}{2} \frac{d W(\tau)}{d \tau} \leq & -\frac{1}{2} \int_{S_{2 l}}^{S_{2 u}} \int_{S_{1 l}}^{S_{1 u}} u^{2}\left\{\frac{\partial}{\partial S_{1}}\left(a_{1}-\lambda_{1} b_{1}-b_{1} \frac{\partial b_{1}}{\partial S_{1}}-\frac{1}{2} \frac{\partial}{\partial S_{2}}\left(\rho b_{1} b_{2}\right)\right)\right. \\
& \left.+\frac{\partial}{\partial S_{2}}\left(a_{2}-\lambda_{2} b_{2}-b_{2} \frac{\partial b_{2}}{\partial S_{2}}-\frac{1}{2} \frac{\partial}{\partial S_{1}}\left(\rho b_{1} b_{2}\right)\right)+2 r\right\} d S_{1} d S_{2} \\
\leq & \frac{1}{2} c_{1} W(\tau) .
\end{aligned}
$$

Therefore, according to the Gronwall inequality, we arrive at

$$
0 \leq W(\tau) \leq e^{c_{1} \tau} W(0) .
$$

Because $W(0)=0$, we get $W(\tau) \equiv 0$. Then, $u \equiv 0$, or $u_{1} \equiv u_{2}$; that is, the solution of $(3.1)$ is unique.

Here we would like to make some remarks. The first one is about the conditions given in the theorem. If $a_{1}, a_{2}, b_{1}, b_{2}, \lambda_{1}, \lambda_{2}, r$, the first derivatives of $a_{1}, a_{2}, \lambda_{1}$ and $\lambda_{2}$, and the first and second derivatives of $\rho, b_{1}$ and $b_{2}$ are bounded, then conditions (2.3), (2.4) are reduced to (2.5), (2.6) respectively, and condition (ii) is always satisfied. The partial differential equation in (3.1) is called a degenerate parabolic partial differential equation because of the equality conditions in (2.3) and (2.4). It is clear that the result can be applied to any degenerate parabolic problems from various fields.

When there are $K$ random variables governed by

$$
d S_{i}=a_{i}\left(S_{1}, S_{2}, \cdots, S_{K}, t\right) d t+b_{i}\left(S_{1}, S_{2}, \cdots, S_{K}, t\right) d X_{i}, \quad i=1,2, \cdots, K,
$$

similar results can still be proven. For the proof above, a key fact we used is $|\rho| \leq 1$, which means that

$$
\left(\begin{array}{ll}
1 & \rho \\
\rho & 1
\end{array}\right)
$$

is semi-positive definite, i.e., all its eigenvalues are greater than or equal to zero. For multi-dimensional cases, we need the fact that the correlation matrix

$$
\left(\begin{array}{cccc}
1 & \rho_{12} & \cdots & \rho_{1 K} \\
\rho_{21} & 1 & \cdots & \rho_{2 K} \\
\vdots & \vdots & \ddots & \vdots \\
\rho_{K 1} & \rho_{K 2} & \cdots & 1
\end{array}\right)
$$

is semi-positive definite. Here $\rho_{i, j}=\mathrm{E}\left[d X_{i} d X_{j}\right] / d t$. 
The meaning of the final-value problem (3.1) having a unique solution is that the solution of (3.1) is completely determined by the final condition. This also means that the random variables will never reach the boundaries if they are inside the domain at the beginning [3]. This is because if the random variables reach the boundaries, then the solution must also be affected by what happens at the boundaries. Therefore, if stochastic models satisfy the reversion conditions, then those random variables should be guaranteed on the finite domain $\left[S_{1 l}, S_{1 u}\right] \times\left[S_{2 l}, S_{2 u}\right]$. When $\left.\frac{\partial b_{i}\left(S_{1}, S_{2}, t\right)}{\partial S_{i}}\right|_{S_{i}=S_{i l}}$ and $\left.\frac{\partial b_{i}\left(S_{1}, S_{2}, t\right)}{\partial S_{i}}\right|_{S_{i}=S_{i u}}$ are bounded, then conditions (2.3) and (2.4) are reduced to (2.5) and (2.6). Under conditions (2.5) and (2.6), the fact that the random variable will never reach the boundaries has been proven for the one dimensional case in [3]. It can be expected that the same result is still true for multi-dimensional cases and when conditions (2.3) and (2.4) cannot be reduced to (2.5) and (2.6).

At present, a stochastic model in finance usually describes a random variable which can take its value on an infinite domain. In many cases, closed-form solutions can be found for such a model. This is an advantage of such a model. However, it seems that assuming a random variable (such as interest rates, volatilities) to be defined on a finite domain and designing a model from market data are more realistic. In this case, we need to require conditions (2.3) and (2.4). Also indeed, condition (2.3) is satisfied by models defined on $[0, \infty)$ and having unique solutions for the corresponding final-value problems. For example, in the popular model [1]

$$
d S=\mu S d t+\sigma S d X
$$

condition (2.3) is satisfied at $S=0$. In the Cox-Ingersoll-Ross interest rate model [2]

$$
d r=(\bar{\mu}-\bar{\gamma} r) d t+\sqrt{\alpha r} d X, \quad \bar{\mu}, \bar{\gamma}, \alpha>0,
$$

condition (2.3) is reduced to $\bar{\mu}-\alpha / 2 \geq 0$ if the lower bound is zero. Actually, this condition is required in order to let $r$ be nonnegative. As will be pointed in the next section, a random variable on $[0, \infty)$ can be transferred to a new random variable on $[0,1)$. When a random variable is transferred to a new random variable on a finite domain, the new final-value problem should still have a unique solution if the original final-value problem does. This means that for these models defined on $[0, \infty)$ and having unique solutions for the corresponding final-value problems, the new random variable must satisfy condition (2.4) at the upper bound of the domain $[0,1]$. Therefore, it can be said that these models satisfy reversion conditions in some sense. For those models defined on $(-\infty, \infty)$ and having unique solutions for their final-value problems, the situation is similar.

Stochastic models satisfying such conditions can describe various stochastic variables, such as stochastic volatilities, stochastic spot interest rates and London Interbank Offer Rates, stock prices, and bond prices. Stochastic spot interest rates and volatilities satisfying such conditions have been applied to derivative security problems with free boundaries ([7] and [6]). In this paper, taking a problem whose corresponding stochastic models satisfy the reversion conditions as an example, we will show that numerical solutions of such final-value problems can be obtained without using any artificial boundary conditions and that if the singularity-separating method and extrapolation techniques are used, then numerical solutions are very good even on quite coarse meshes. 


\section{Uniqueness of Solutions for European Options on Assets with Stochastic Volatilities}

In this section, we consider a special two-factor financial derivative: options on assets with stochastic volatilities. We assume that the asset price $S$ follows the following stochastic process

$$
d S=\mu S d t+\sigma S d X_{1}, \quad 0 \leq S
$$

and that the volatility $\sigma$ is also a random variable, and its evolution is governed by

$$
d \sigma=p(\sigma, t) d t+q(\sigma, t) d X_{2}, \quad \sigma_{l} \leq \sigma \leq \sigma_{u},
$$

where the two random increments $d X_{1}$ and $d X_{2}$ are two Wiener processes. $d X_{1}$ and $d X_{2}$ are correlated and $\mathrm{E}\left[d X_{1} d X_{2}\right]=\rho d t$. Furthermore, we assume that the stochastic model for $\sigma$ satisfies reversion conditions; that is, the following relations hold:

$$
\left\{\begin{array}{l}
p\left(\sigma_{l}, t\right)-q\left(\sigma_{l}, t\right) \frac{\partial q}{\partial \sigma}\left(\sigma_{l}, t\right) \geq 0, \\
q\left(\sigma_{l}, t\right)=0
\end{array}\right.
$$

and

$$
\left\{\begin{array}{l}
p\left(\sigma_{u}, t\right)-q\left(\sigma_{u}, t\right) \frac{\partial q}{\partial \sigma}\left(\sigma_{u}, t\right) \leq 0 \\
q\left(\sigma_{u}, t\right)=0
\end{array}\right.
$$

or when $\frac{\partial q}{\partial \sigma}\left(\sigma_{l}, t\right)$ and $\frac{\partial q}{\partial \sigma}\left(\sigma_{u}, t\right)$ are bounded,

$$
\left\{\begin{array}{l}
p\left(\sigma_{l}, t\right) \geq 0 \\
q\left(\sigma_{l}, t\right)=0
\end{array}\right.
$$

and

$$
\left\{\begin{array}{l}
p\left(\sigma_{u}, t\right) \leq 0 \\
q\left(\sigma_{u}, t\right)=0
\end{array}\right.
$$

hold. Suppose that $V(S, \sigma, t)$ is the value of such an option. $V(S, \sigma, t)$ satisfies

$$
\begin{gathered}
\frac{\partial V}{\partial t}+\frac{1}{2} \sigma^{2} S^{2} \frac{\partial^{2} V}{\partial S^{2}}+\rho \sigma q S \frac{\partial^{2} V}{\partial S \partial \sigma}+\frac{1}{2} q^{2} \frac{\partial^{2} V}{\partial \sigma^{2}} \\
+\left(r-D_{0}\right) S \frac{\partial V}{\partial S}+(p-\lambda q) \frac{\partial V}{\partial \sigma}-r V=0 .
\end{gathered}
$$

This equation holds for $S \in[0, \infty)$. In order to convert the problem on an infinite domain into one on a finite domain, we introduce the following transformation:

$$
\left\{\begin{aligned}
\xi & =\frac{S}{S+P_{m}}, \\
\sigma & =\sigma \\
t & =t \\
\bar{V} & =\frac{V}{S+P_{m}}
\end{aligned}\right.
$$


where $P_{m}$ is a positive constant. Since there are the following expressions:

$$
\begin{array}{ll}
S=\frac{\xi P_{m}}{1-\xi}, & S+P_{m}=\frac{P_{m}}{1-\xi}, \\
\frac{d \xi}{d S}=\frac{(1-\xi)^{2}}{P_{m}}, & \frac{\partial V}{\partial t}=\frac{P_{m}}{1-\xi} \frac{\partial \bar{V}}{\partial t}, \\
\frac{\partial V}{\partial S}=\bar{V}+(1-\xi) \frac{\partial \bar{V}}{\partial \xi}, & \frac{\partial V}{\partial \sigma}=\frac{P_{m}}{1-\xi} \frac{\partial \bar{V}}{\partial \sigma}, \\
\frac{\partial^{2} V}{\partial S^{2}}=\frac{(1-\xi)^{3}}{P_{m}} \frac{\partial^{2} \bar{V}}{\partial \xi^{2}}, & \frac{\partial^{2} V}{\partial S \partial \sigma}=\frac{\partial \bar{V}}{\partial \sigma}+(1-\xi) \frac{\partial^{2} \bar{V}}{\partial \xi \partial \sigma}, \\
\frac{\partial^{2} V}{\partial \sigma^{2}}=\frac{P_{m}}{1-\xi} \frac{\partial^{2} \bar{V}}{\partial \sigma^{2}}, &
\end{array}
$$

(4.7) can be rewritten as

$$
\begin{aligned}
& \frac{\partial \bar{V}}{\partial t}+\frac{1}{2} \sigma^{2} \xi^{2}(1-\xi)^{2} \frac{\partial^{2} \bar{V}}{\partial \xi^{2}}+\rho \sigma \xi(1-\xi) q \frac{\partial^{2} \bar{V}}{\partial \xi \partial \sigma}+\frac{1}{2} q^{2} \frac{\partial^{2} \bar{V}}{\partial \sigma^{2}}+\left(r-D_{0}\right) \xi(1-\xi) \frac{\partial \bar{V}}{\partial \xi} \\
& +(p-(\lambda-\rho \sigma \xi) q) \frac{\partial \bar{V}}{\partial \sigma}-\left(r-\left(r-D_{0}\right) \xi\right) \bar{V}=0 .
\end{aligned}
$$

Since the transformation above converts a value of $S \in[0, \infty)$ into a value of $\xi \in[0,1]$, $\bar{V}(\xi, \sigma, t)$ is defined on the domain $[0,1] \times\left[\sigma_{l}, \sigma_{u}\right] \times[0, T]$. Therefore, the determination of European option prices in this case reduces to finding the solution of the following final-value problem:

$$
\left\{\begin{array}{l}
\frac{\partial \bar{V}}{\partial t}+\frac{1}{2} \sigma^{2} \xi^{2}(1-\xi)^{2} \frac{\partial^{2} \bar{V}}{\partial \xi^{2}}+\rho \sigma \xi(1-\xi) q \frac{\partial^{2} \bar{V}}{\partial \xi \partial \sigma}+\frac{1}{2} q^{2} \frac{\partial^{2} \bar{V}}{\partial \sigma^{2}}+\left(r-D_{0}\right) \xi(1-\xi) \frac{\partial \bar{V}}{\partial \xi} \\
+(p-(\lambda-\rho \sigma \xi) q) \frac{\partial \bar{V}}{\partial \sigma}-\left(r-\left(r-D_{0}\right) \xi\right) \bar{V}=0, \\
\quad \xi \in[0,1], \quad \sigma \in\left[\sigma_{l}, \sigma_{u}\right], \quad t \in[0, T], \\
\bar{V}(\xi, \sigma, T)=f(\xi, \sigma), \quad \xi \in[0,1], \quad \sigma \in\left[\sigma_{l}, \sigma_{u}\right] .
\end{array}\right.
$$

This problem is in the form of (3.1) with

$$
\begin{aligned}
& a_{1}=\left(r-D_{0}\right) \xi(1-\xi)+\lambda \sigma \xi(1-\xi), \\
& b_{1}=\sigma \xi(1-\xi) \\
& a_{2}=p(\sigma, t)+\rho \sigma \xi q(\sigma, t) \\
& b_{2}=q(\sigma, t)
\end{aligned}
$$

and the coefficient of $\bar{V}$ here is $-\left(r-\left(r-D_{0}\right) \xi\right)$. In order to have a unique solution, the key is that $a_{1}, b_{1}, a_{2}$ and $b_{2}$ should satisfy the reversion conditions (2.3) and (2.4). In this case, $a_{1}$ and $b_{1}$ always satisfy (2.3) and (2.4). That $a_{2}$ and $b_{2}$ satisfy the reversion conditions is equivalent to fulfillment of (4.3) and (4.4). Therefore, if (4.3), (4.4), conditions (ii) and (iii) of Theorem 1 are satisfied, then (4.9) has a unique solution. 


\section{Examples}

Here, examples are given to explain (i) if conditions (4.3) and (4.4) hold, then we can solve problem (4.9) numerically by using the partial differential equation and final condition only; that is, without using any artificial conditions, and (ii) if the singularity-separating method and extrapolation techniques are used, then very good results can be obtained even on very coarse meshes.

Consider a two-factor European vanilla call option problem and let its value be $c(S, \sigma, t)$. Suppose that the volatility model satisfies the reversion conditions. Then no boundary conditions need to be given at the boundaries $\sigma=\sigma_{l}$ and $\sigma=\sigma_{u}$. Therefore, the value of the two-factor European vanilla call option is the solution of the following final-value problem:

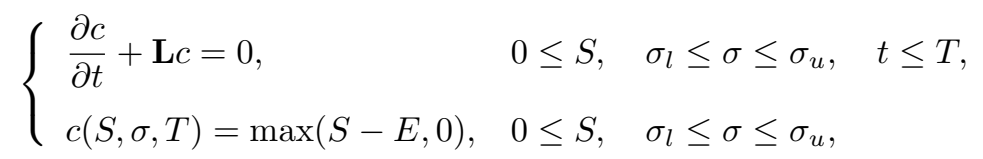

where

$$
\mathbf{L}=\frac{1}{2} \sigma^{2} S^{2} \frac{\partial^{2}}{\partial S^{2}}+\rho \sigma S q \frac{\partial^{2}}{\partial S \partial \sigma}+\frac{1}{2} q^{2} \frac{\partial^{2}}{\partial \sigma^{2}}+\left(r-D_{0}\right) S \frac{\partial}{\partial S}+(p-\lambda q) \frac{\partial}{\partial \sigma}-r
$$

and $E$ is the exercise price of the call option. In order to make numerical methods more efficient, we introduce

$$
\bar{c}(S, \sigma, t)=c(S, \sigma, t)-c_{0}(S, \sigma, t) .
$$

Here $c_{0}(S, \sigma, t)$ is the price of a one-factor European vanilla call option; that is, the price of the European vanilla call option with a constant $\sigma$. Its value is given by (see [1], [8])

$$
c_{0}(S, \sigma, t)=S e^{-D_{0}(T-t)} N\left(d_{1}\right)-E e^{-r(T-t)} N\left(d_{2}\right)
$$

where

$$
\begin{aligned}
N(z) & =\frac{1}{\sqrt{2 \pi}} \int_{-\infty}^{z} e^{-\xi^{2} / 2} d \xi \\
d_{1} & =\left[\ln \frac{S e^{-D_{0}(T-t)}}{E e^{-r(T-t)}}+\frac{1}{2} \sigma^{2}(T-t)\right] /(\sigma \sqrt{T-t}), \\
d_{2} & =d_{1}-\sigma \sqrt{T-t} .
\end{aligned}
$$

Since $c_{0}(S, \sigma, t)$ satisfies the Black-Scholes equation, the difference $\bar{c}$ is the solution of the following final-value problem:

$$
\begin{cases}\frac{\partial \bar{c}}{\partial t}+\mathbf{L} \bar{c}=f(S, \sigma, t), & 0 \leq S, \quad \sigma_{l} \leq \sigma \leq \sigma_{u}, \quad 0 \leq t \leq T \\ \bar{c}(S, \sigma, T)=0, & 0 \leq S, \quad \sigma_{l} \leq \sigma \leq \sigma_{u}\end{cases}
$$

where

$$
f(S, \sigma, t)=-\rho \sigma S q \frac{\partial^{2} c_{0}}{\partial S \partial \sigma}-\frac{1}{2} q^{2} \frac{\partial^{2} c_{0}}{\partial \sigma^{2}}-(p-\lambda q) \frac{\partial c_{0}}{\partial \sigma} .
$$


From the expressions of $c_{0}(S, \sigma, t)$, noticing

$$
\begin{aligned}
\frac{\partial c_{0}}{\partial S} & =e^{-D_{0}(T-t)} N\left(d_{1}\right), \\
\frac{\partial d_{1}}{\partial \sigma} & =\sqrt{T-t}-\left[\ln \frac{S e^{-D_{0}(T-t)}}{E e^{-r(T-t)}}+\frac{1}{2} \sigma^{2}(T-t)\right] /\left(\sigma^{2} \sqrt{T-t}\right) \\
& =\sqrt{T-t}-\frac{d_{1}}{\sigma}, \\
\frac{\partial d_{2}}{\partial \sigma} & =\frac{\partial d_{1}}{\partial \sigma}-\sqrt{T-t}=-\frac{d_{1}}{\sigma}, \\
N^{\prime}(z) & =\frac{1}{\sqrt{2 \pi}} e^{-z^{2} / 2},
\end{aligned}
$$

we can easily find

$$
\begin{aligned}
\frac{\partial c_{0}}{\partial \sigma} & =S e^{-D_{0}(T-t)} N^{\prime}\left(d_{1}\right) \frac{\partial d_{1}}{\partial \sigma}-E e^{-r(T-t)} N^{\prime}\left(d_{2}\right) \frac{\partial d_{2}}{\partial \sigma} \\
& =S \sqrt{T-t} e^{-D_{0}(T-t)} N^{\prime}\left(d_{1}\right), \\
\frac{\partial^{2} c_{0}}{\partial \sigma^{2}} & =S \sqrt{T-t} e^{-D_{0}(T-t)} N^{\prime \prime}\left(d_{1}\right) \frac{\partial d_{1}}{\partial \sigma} \\
& =-S \sqrt{T-t} e^{-D_{0}(T-t)} d_{1} N^{\prime}\left(d_{1}\right) \frac{\partial d_{1}}{\partial \sigma} \\
\frac{\partial^{2} c_{0}}{\partial S \partial \sigma} & =e^{-D_{0}(T-t)} N^{\prime}\left(d_{1}\right) \frac{\partial d_{1}}{\partial \sigma} .
\end{aligned}
$$

As we see from (5.1), the derivative of $c(S, \sigma, t)$ with respect to $S$ is discontinuous at $t=T$ and $S=E$. However, (5.4) shows that at $t=T$ the derivative of $\bar{c}(S, \sigma, t)$ with respect to $S$ is identically zero. Therefore, when a numerical method is used, the truncation error for problem (5.4) will be much smaller than that of problem (5.1). In what follows, we will refer to such a technique as the singularity-separating method (SSM), since the original weak singularity has been separated.

The final-value problem (5.4) is defined on an infinite domain. In order to convert this problem into an initial-value problem on a finite domain, let $\xi$ be defined as in (4.8) with $P_{m}=E$, and define

$$
\tau=T-t, \quad u(\xi, \sigma, \tau)=\frac{\bar{c}(S, \sigma, t)}{S+E} .
$$

In the $\{\xi, \sigma, \tau\}$-space, problem (5.4) becomes

$$
\left\{\begin{array}{c}
\frac{\partial u}{\partial \tau}=a_{1} \frac{\partial^{2} u}{\partial \xi^{2}}+a_{2} \frac{\partial^{2} u}{\partial \xi \partial \sigma}+a_{3} \frac{\partial^{2} u}{\partial \sigma^{2}}+a_{4} \frac{\partial u}{\partial \xi}+a_{5} \frac{\partial u}{\partial \sigma}+a_{6} u+a_{7} \\
0 \leq \xi \leq 1, \quad \sigma_{l} \leq \sigma \leq \sigma_{u}, \quad 0 \leq \tau \leq T \\
u(\xi, \sigma, 0)=0,0 \leq \xi \leq 1, \quad \sigma_{l} \leq \sigma \leq \sigma_{u}
\end{array}\right.
$$


where

$$
\begin{aligned}
a_{1}= & \frac{1}{2} \sigma^{2} \xi^{2}(1-\xi)^{2} \\
a_{2}= & \rho \sigma \xi(1-\xi) q \\
a_{3}= & \frac{1}{2} q^{2} \\
a_{4}= & \left(r-D_{0}\right) \xi(1-\xi) \\
a_{5}= & p-(\lambda-\rho \sigma \xi) q \\
a_{6}= & -\left[r(1-\xi)+D_{0} \xi\right] \\
a_{7}= & -f(\xi E /(1-\xi), \sigma, T-\tau)(1-\xi) / E \\
= & \rho \sigma \xi q e^{-D_{0}(T-t)} N^{\prime}\left(d_{1}\right) \frac{\partial d_{1}}{\partial \sigma}-\frac{1}{2} q^{2} \xi \sqrt{T-t} e^{-D_{0}(T-t)} d_{1} N^{\prime}\left(d_{1}\right) \frac{\partial d_{1}}{\partial \sigma} \\
& +(p-\lambda q) \xi \sqrt{T-t} e^{-D_{0}(T-t)} N^{\prime}\left(d_{1}\right) \\
= & \frac{1}{\sqrt{2 \pi}} \xi e^{-D_{0} \tau-d_{1}^{2} / 2}\left[q\left(\sqrt{\tau}-d_{1} / \sigma\right)\left(\rho \sigma-q \sqrt{\tau} d_{1} / 2\right)+(p-\lambda q) \sqrt{\tau}\right] .
\end{aligned}
$$

Once we have a solution of (5.6), $u(\xi, \sigma, \tau)$, we can get the value of the two-factor European call option by

$$
\begin{aligned}
c(S, \sigma, t) & =\bar{c}(S, \sigma, t)+c_{0}(S, \sigma, t) \\
& =(S+E) u\left(\frac{S}{S+E}, \sigma, T-t\right)+c_{0}(S, \sigma, t) .
\end{aligned}
$$

We calculate the solution of problem (5.6) by the following method. Let $u_{m, i}^{n}$ be the approximate value of $u$ at $\xi=m \Delta \xi, \sigma=\sigma_{l}+i \Delta \sigma$ and $\tau=n \Delta \tau$, where $\Delta \xi=1 / M$, $\Delta \sigma=\left(\sigma_{u}-\sigma_{l}\right) / I$ and $\Delta \tau=1 / N, M, I, N$ being integers. This partial differential equation can be discretized by the following scheme. If $\sigma \neq \sigma_{l}$ and $\sigma \neq \sigma_{u}$, then at a point $\left(\xi_{m}, \sigma_{i}, \tau^{n+1 / 2}\right)$ the partial differential equation in (5.6) can be discretized by the following second order approximation:

$$
\begin{aligned}
\frac{u_{m, i}^{n+1}-u_{m, i}^{n}}{\Delta \tau}= & \frac{a_{1}}{2 \Delta \xi^{2}}\left(u_{m+1, i}^{n+1}-2 u_{m, i}^{n+1}+u_{m-1, i}^{n+1}+u_{m+1, i}^{n}-2 u_{m, i}^{n}+u_{m-1, i}^{n}\right) \\
& +\frac{a_{2}}{8 \Delta \sigma \Delta \xi}\left(u_{m+1, i+1}^{n+1}-u_{m+1, i-1}^{n+1}-u_{m-1, i+1}^{n+1}+u_{m-1, i-1}^{n+1}\right. \\
& \left.\quad+u_{m+1, i+1}^{n}-u_{m+1, i-1}^{n}-u_{m-1, i+1}^{n}+u_{m-1, i-1}^{n}\right) \\
& +\frac{a_{3}}{2 \Delta \sigma^{2}}\left(u_{m, i+1}^{n+1}-2 u_{m, i}^{n+1}+u_{m, i-1}^{n+1}+u_{m, i+1}^{n}-2 u_{m, i}^{n}+u_{m, i-1}^{n}\right) \\
& +\frac{a_{4}}{4 \Delta \xi}\left(u_{m+1, i}^{n+1}-u_{m-1, i}^{n+1}+u_{m+1, i}^{n}-u_{m-1, i}^{n}\right) \\
& +\frac{a_{5}}{4 \Delta \sigma}\left(u_{m, i+1}^{n+1}-u_{m, i-1}^{n+1}+u_{m, i+1}^{n}-u_{m, i-1}^{n}\right) \\
& +\frac{a_{6}}{2}\left(u_{m, i}^{n+1}+u_{m, i}^{n}\right)+a_{7}, \\
& \quad m=0,1, \cdots, M, \quad i=1,2, \cdots, I-1
\end{aligned}
$$

Here all the coefficients $a_{1}-a_{7}$ should be evaluated at the point $\left(\xi_{m}, \sigma_{i}, \tau^{n+1 / 2}\right)$ in order to guarantee second order accuracy. 
At the boundaries $\sigma=\sigma_{l}$ and $\sigma=\sigma_{u}$, due to $q=0$ the partial differential equation in (5.6) becomes a hyperbolic equation with respect to $\sigma$ :

$$
\frac{\partial u}{\partial \tau}=a_{1} \frac{\partial^{2} u}{\partial \xi^{2}}+a_{4} \frac{\partial u}{\partial \xi}+a_{5} \frac{\partial u}{\partial \sigma}+a_{6} u+a_{7}
$$

From the reversion conditions, we see that $a_{5}=p-(\lambda-\rho \sigma \xi) q=p \geq p-q \partial q / \partial \sigma \geq$ $0^{4)}$ at the boundary $\sigma=\sigma_{l}$ and $a_{5}=p-(\lambda-\rho \sigma \xi) q=p \leq p-q \partial q / \partial \sigma \leq 0$ at $\sigma=\sigma_{u}$. These facts tell us that the value of $u$ on the boundaries $\sigma=\sigma_{l}$ and $\sigma=\sigma_{u}$ should be determined by the value of $u$ inside the domain. Hence, we can approximate the partial differential equation in (5.6) at the boundaries $\sigma=\sigma_{l}$ and $\sigma=\sigma_{u}$ by

$$
\begin{aligned}
\frac{u_{m, 0}^{n+1}-u_{m, 0}^{n}}{\Delta \tau}= & \frac{a_{1}}{2 \Delta \xi^{2}}\left(u_{m+1,0}^{n+1}-2 u_{m, 0}^{n+1}+u_{m-1,0}^{n+1}+u_{m+1,0}^{n}-2 u_{m, 0}^{n}+u_{m-1,0}^{n}\right) \\
& +\frac{a_{4}}{4 \Delta \xi}\left(u_{m+1,0}^{n+1}-u_{m-1,0}^{n+1}+u_{m+1,0}^{n}-u_{m-1,0}^{n}\right) \\
& +\frac{a_{5}}{4 \Delta \sigma}\left(-u_{m, 2}^{n+1}+4 u_{m, 1}^{n+1}-3 u_{m, 0}^{n+1}-u_{m, 2}^{n}+4 u_{m, 1}^{n}-3 u_{m, 0}^{n}\right) \\
& +\frac{a_{6}}{2}\left(u_{m, 0}^{n+1}+u_{m, 0}^{n}\right)+a_{7}, \\
& m=0,1, \cdots, M
\end{aligned}
$$

and

$$
\begin{aligned}
\frac{u_{m, I}^{n+1}-u_{m, I}^{n}}{\Delta \tau}= & \frac{a_{1}}{2 \Delta \xi^{2}}\left(u_{m+1, I}^{n+1}-2 u_{m, I}^{n+1}+u_{m-1, I}^{n+1}+u_{m+1, I}^{n}-2 u_{m, I}^{n}+u_{m-1, I}^{n}\right) \\
& +\frac{a_{4}}{4 \Delta \xi}\left(u_{m+1, I}^{n+1}-u_{m-1, I}^{n+1}+u_{m+1, I}^{n}-u_{m-1, I}^{n}\right) \\
& +\frac{a_{5}}{4 \Delta \sigma}\left(3 u_{m, I}^{n+1}-4 u_{m, I-1}^{n+1}+u_{m, I-2}^{n+1}+3 u_{m, I}^{n}-4 u_{m, I-1}^{n}+u_{m, I-2}^{n}\right) \\
& +\frac{a_{6}}{2}\left(u_{m, I}^{n+1}+u_{m, I}^{n}\right)+a_{7}, \\
& m=0,1, \cdots, M
\end{aligned}
$$

respectively. Here $\partial u / \partial \sigma$ is discretized by a one-sided second order scheme in order for all the node points involved to be in the computational domain, and $a_{1}$ and $a_{4}-a_{7}$ are also evaluated at the point $\left(\xi_{m}, \sigma_{i}, \tau^{n+1 / 2}\right), i=0$ or $I$. When $u_{m, i}^{n}$, $m=0,1, \cdots, M, i=0,1, \cdots, I$ are known, from (5.8)-(5.10) we can determine $u_{m, i}^{n+1}$, $m=0,1, \cdots, M, i=0,1, \cdots, I$. The initial condition gives $u_{m, i}^{0}, m=0,1, \cdots, M$, $i=0,1, \cdots, I$. Therefore, we can do this procedure for $n=0,1, \cdots, N-1$ successively and finally find $u_{m, i}^{N}, m=0,1, \cdots, M, i=0,1, \cdots, I$. Since truncation errors are second order everywhere and the solutions are smooth, it can be expected that the error is $O\left(\Delta \xi^{2}, \Delta \tau^{2}\right)$ and extrapolation techniques will be very useful (see [5]).

In Figure 5.1, the price of a European call option obtained in this way is plotted. The mesh used is $20 \times 20 \times 20$, where the first, second and third numbers are $M$, $I$ and $N$ respectively. The parameters of the problem are $E=50, T=1, r=0.1$,

4) Since $q^{2} \geq 0$ on the interval $\left[\sigma_{l}, \sigma_{u}\right]$, the derivative $\partial q^{2} / \partial \sigma=2 q \partial q / \partial \sigma$ must be greater than or equal to zero at the lower boundary $\sigma=\sigma_{l}$ and less than or equal to zero at the upper boundary $\sigma=\sigma_{u}$. 


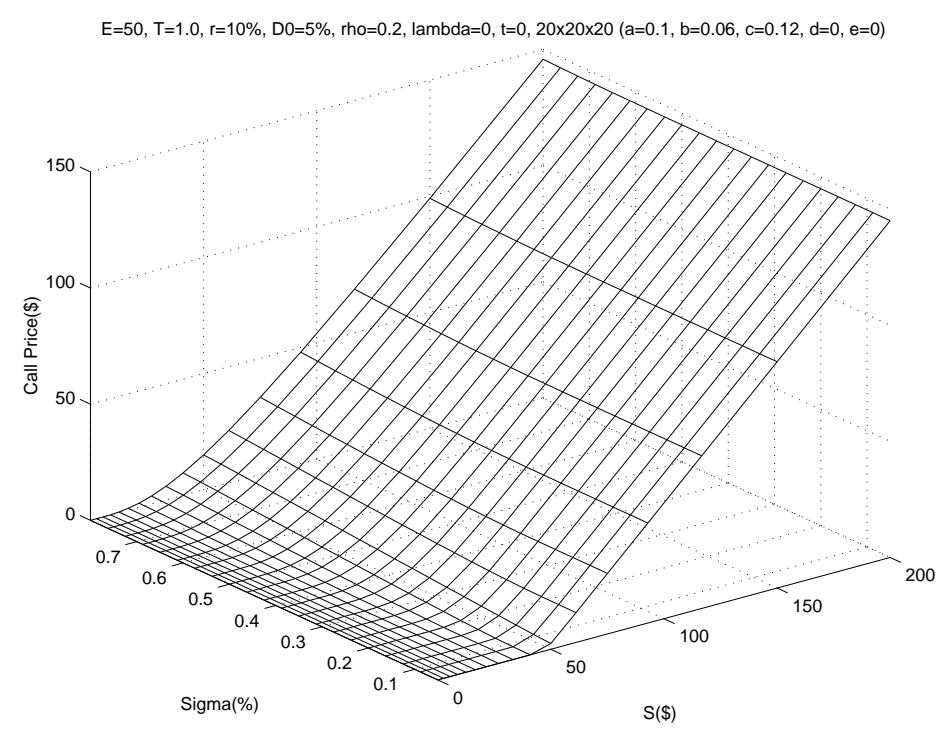

FIG. 5.1. Price of a two-factor European call option

$D_{0}=0.05, t=0$ and the parameter functions are

$$
\begin{aligned}
p & =a(b-\sigma), \quad \sigma_{l} \leq \sigma \leq \sigma_{u}, \\
q & =c \frac{1-\left(1-2 \frac{\sigma-\sigma_{l}}{\sigma_{u}-\sigma_{l}}\right)^{2}}{1-0.975\left(1-2 \frac{\sigma-\sigma_{l}}{\sigma_{u}-\sigma_{l}}\right)^{2}} \sigma, \quad \sigma_{l} \leq \sigma \leq \sigma_{u}, \\
& \rho=0.2, \\
\lambda=d+e \sigma, \quad \sigma_{l} \leq \sigma \leq \sigma_{u}, &
\end{aligned}
$$

where $a=0.1, b=0.06, c=0.12, d=0, e=0, \sigma_{l}=0.05$ and $\sigma_{u}=0.8$. It is easy to check that the reversion conditions are satisfied for $p$ and $q$ here.

When the singularity-separating technique is not adopted, the scheme above can also be used. In that case,

$$
a_{7}=0 \quad \text { and } \quad u(\xi, \sigma, 0)=\max (2 \xi-1,0) .
$$

In order to give some idea about the performance of the method described in this section, we list the values of the option obtained by the method here and by the same difference method without using singularity-separating in Tables 5.1 and 5.2 for $S=50$ and $\sigma=0.2$. From the tables, we see that the exact solution up to the sixth decimal place is 4.848069 , which we obtain by a very fine mesh. Therefore, we can find out the errors of the results up to the sixth decimal place, which are also listed there. Table 5.1 shows that for a $20 \times 20 \times 20$ mesh with extrapolation, the error relative to $E$ is $0.0047 / 50 \approx 10^{-4}$ and the error relative to the option value is $0.0047 / 4.848069 \approx 10^{-3}$. In practice, requiring such an accuracy is reasonable. The 
TABLE 5.1. SSM with and without extrapolation

$$
\begin{gathered}
S=50, E=50, T=1, \sigma=0.2, r=0.1, D_{0}=0.05, \\
a=0.1, b=0.06, c=0.12, d=0, e=0 . \\
\quad \text { Exact solution is } 4.848069 \cdots .
\end{gathered}
$$

\begin{tabular}{|c|c|c|c|c|}
\hline & \multicolumn{2}{|c|}{ Without Extrapolation } & \multicolumn{2}{c|}{ With Extrapolation } \\
\hline Meshes & Solution & $\mid$ Errors $\mid$ & Solution & $\mid$ Errors $\mid$ \\
\hline $10 \times 10 \times 10$ & 4.8143085 & 0.033761 & - & - \\
\hline $20 \times 20 \times 20$ & 4.8361039 & 0.011966 & 4.8433691 & 0.004700 \\
\hline $40 \times 40 \times 40$ & 4.8460151 & 0.002054 & 4.8493188 & 0.001249 \\
\hline $80 \times 80 \times 80$ & 4.8476154 & 0.000454 & 4.8481488 & 0.000079 \\
\hline $160 \times 160 \times 160$ & 4.8479592 & 0.000110 & 4.8480738 & 0.000004 \\
\hline $320 \times 320 \times 320$ & 4.8480421 & 0.000027 & 4.8480697 & less than $10^{-6}$ \\
\hline $640 \times 640 \times 640$ & 4.8480626 & 0.000007 & 4.8480694 & less than $10^{-6}$ \\
\hline $960 \times 960 \times 960$ & 4.8480664 & 0.000003 & 4.8480694 & less than $10^{-6}$ \\
\hline
\end{tabular}

TABLE 5.2. Implicit Method with and without extrapolation

$$
\begin{gathered}
S=50, E=50, T=1, \sigma=0.2, r=0.1, D_{0}=0.05, \\
a=0.1, b=0.06, c=0.12, d=0 \text { and } e=0 . \\
\text { Exact solution is } 4.848069 \cdots .
\end{gathered}
$$

\begin{tabular}{|c|c|c|c|c|}
\hline & \multicolumn{2}{|c|}{ Without Extrapolation } & \multicolumn{2}{c|}{ With Extrapolation } \\
\hline Meshes & Solution & $\mid$ Errors $\mid$ & Solution & $\mid$ Errors $\mid$ \\
\hline $10 \times 10 \times 10$ & 3.1774889 & 1.670580 & - & - \\
\hline $20 \times 20 \times 20$ & 4.2406270 & 0.607442 & 4.5950063 & 0.253063 \\
\hline $40 \times 40 \times 40$ & 4.7179697 & 0.130100 & 4.8770840 & 0.029015 \\
\hline $80 \times 80 \times 80$ & 4.8171183 & 0.030951 & 4.8501678 & 0.002098 \\
\hline $160 \times 160 \times 160$ & 4.8404088 & 0.007661 & 4.8481722 & 0.000103 \\
\hline $320 \times 320 \times 320$ & 4.8461590 & 0.001910 & 4.8480758 & 0.000006 \\
\hline $640 \times 640 \times 640$ & 4.8475923 & 0.000477 & 4.8480700 & 0.000001 \\
\hline $960 \times 960 \times 960$ & 4.8478575 & 0.000212 & 4.8480697 & less than $10^{-6}$ \\
\hline
\end{tabular}

CPU time on a computer with chips Pentium III $800 \mathrm{mHZ}$ is 0.07 seconds. If the singularity-separating technique is not used, in order to reach a similar accuracy, a mesh close to $80 \times 80 \times 80$ should be used and the CPU time is about 8 seconds.

Noticing

$$
\frac{\partial p_{0}}{\partial \sigma}=\frac{\partial c_{0}}{\partial \sigma}, \quad \frac{\partial^{2} p_{0}}{\partial \sigma^{2}}=\frac{\partial^{2} c_{0}}{\partial \sigma^{2}}, \quad \frac{\partial^{2} p_{0}}{\partial S \partial \sigma}=\frac{\partial^{2} c_{0}}{\partial S \partial \sigma},
$$

we see that the difference between the two-factor and one-factor put options is also the solution of (5.6). Therefore, in order to have the price of a European put option, we can first solve (5.6) and then obtain the put price by

$$
p(S, \sigma, t)=(S+E) u\left(\frac{S}{S+E}, \sigma, T-t\right)+p_{0}(S, \sigma, t)
$$

where $p_{0}(S, \sigma, t)$ is the price of the one-factor put option (for its concrete expression, see [8]). In Figure 5.2, the price of a two-factor European put option obtained by this 


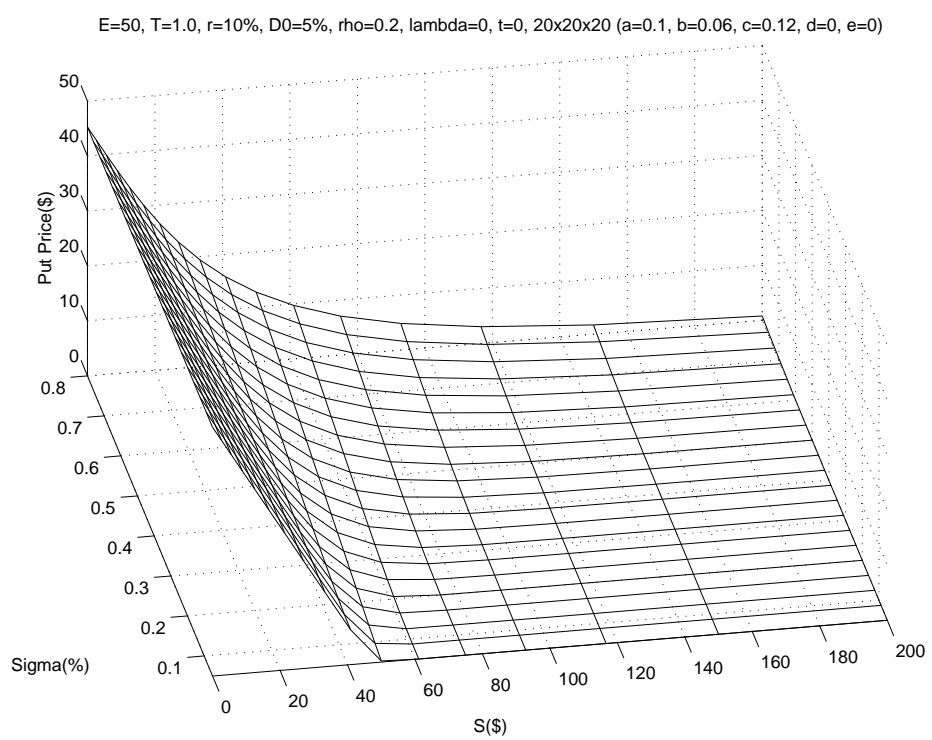

FIG. 5.2. Price of a two-factor European put option

way is shown. The parameters of the problem and the parameter functions are the same as those for the two-factor European call option.

Finally, we give an example to explain that if the reversion conditions are not satisfied, then the final-value problem (4.9) is not well-posed and we cannot determine the solution using only the partial differential equation and the final condition in (4.9). Consider a problem with $a=1, b=0, c=0.012, d=0, e=0$ and $T=2$. The other parameters are the same as before. We still use the numerical method above to find the numerical solution. In Figure 5.3, we give the variation of $u$ with $S$ on the line $\sigma=\sigma_{l}$ at time $t=0$. From there, we can see some "non-physical" oscillations. This shows that for this case the solution is not determined only by the partial differential equation and final condition. The reason is that a proper boundary condition is needed at the boundary $\sigma=\sigma_{l}$ because the inequality condition of (4.5) is not satisfied at $\sigma=\sigma_{l}$ due to $b=0<\sigma_{l}=0.05$. If a reasonable condition cannot be given, then an artificial boundary condition has to be added. If the artificial boundary condition is not proper, then one will encounter some difficulty during computation.

\section{Conclusion}

In this paper, we introduce reversion conditions for stochastic models. Also we prove that if the models satisfy reversion conditions, then the final-value problem of general two-factor financial derivative equations has a unique solution. For such problems, we can obtain their numerical solutions without using any artificial conditions. Examples show that if the singularity-separating method and extrapolation techniques are used, then very good solutions can be obtained even on very coarse meshes. 


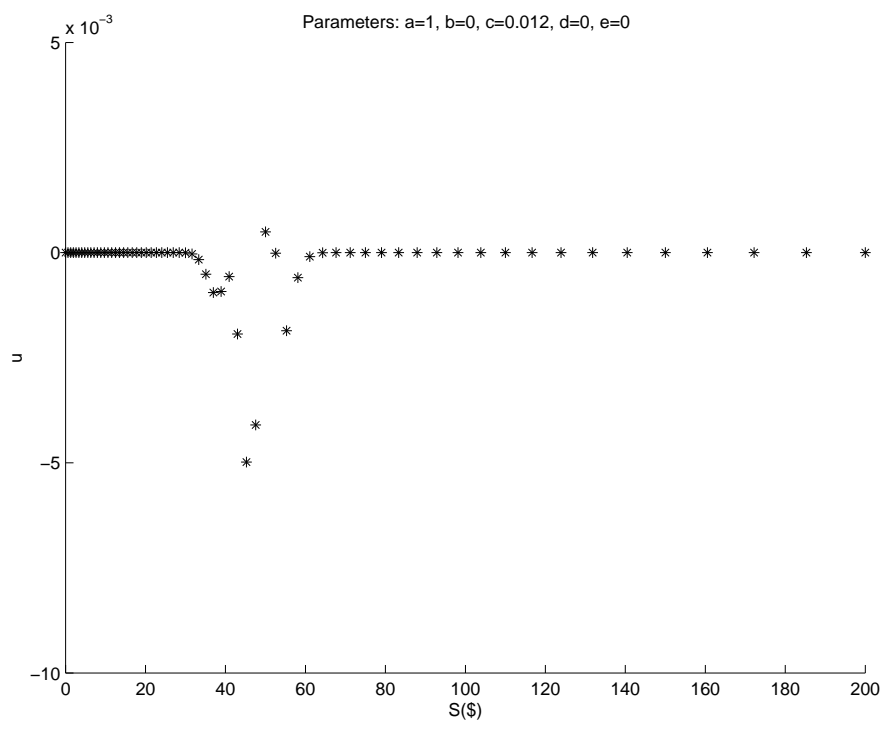

FIG. 5.3. Variation of $u$ with $S$ on the line $\sigma=0.05$ at $t=0$.

$$
\left(E=50, T=2, r=10 \%, D_{0}=5 \%, 80 \times 40 \times 80\right)
$$

\section{REFERENCES}

[1] F. Black and M. Scholes, The valuation of options and corporate liabilities. J. Poli. Eco., 81:637654, 1973.

[2] J.C. Cox, J.E. Ingersoll, and S.A. Ross A theory of the term structure of interest rates. Econometrica, 53:385-407,1985.

[3] I.I. Gihman and A.N. Skorohod, Stochastic Differential Equations. Springer-Verlag, New York, 1972.

[4] M.D. Marcozzi, S. Choi, and C.S. Chen, On the use of boundary conditions for variational formulations arising in financial mathematics. Appl. Math. Comp., 124:197-214, 2001.

[5] Z.Z. Sun, N.N. Yang, and Y.L. Zhu, Convergence of Second-Order Difference Schemes and Extrapolation Algorithm for Degenerate Parabolic Equations. Working paper, University of North Carolina at Charlotte, 2001.

[6] Y.L. Zhu and J. Li, Numerical solutions for american options on assets with stochastic volatilities. Working Paper, University of North Carolina at Charlotte, 2001.

[7] Y.L. Zhu and Y. Sun, The singularity-separating methods for two-factor convertible bonds. J. Comp. Finance., 3(1):91-110, 1999.

[8] Y.L. Zhu, X. Wu, and I.L. Chern, Derivatives Securities and Finite Difference Methods. University of North Carolina at Charlotte, 2002. 